\title{
Erythema Multiforme-like Dermatitis Due to Isoniazid Hypersensitivity in a Patient With Psoriasis
}

\author{
Ayşe Baççıŏlu, MD; Ayşe Anıl Karabulut, MD; Ayşe Füsun Kalpaklığlu, MD
}

\section{PRACTICE POINTS}

- Hypersensitivity skin reactions to antituberculosis (TB) drugs are on the rise due to the increasing use of anti-tumor necrosis factor $\alpha$. Isoniazid (INH) use will be more prevalent than in the past for the treatment of latent TB.

- Even though the skin-restricted adverse events to $\mathrm{INH}$ are rare and minor, particular attention should be paid to patients with dermatologic diseases such as psoriasis.

To the Editor:

Psoriasis vulgaris is a chronic autoimmune inflammatory disease and biologic agents, such as anti-tumor necrosis factor $\alpha$ (TNF- $\alpha$ ), are alternative drugs in case of resistance or adverse events to conventional ones. ${ }^{1}$ The limitation of these agents is immunosuppression that may cause infections such as tuberculosis (TB). Prophylaxis is indicated to latent TB diseases if the purified protein derivative (tuberculin) skin test is higher than $5 \mathrm{~mm}$ before starting these treatments. The challenge in TB treatment is adverse drug reactions (ADRs) that are reported in $4 \%$ to $6 \%$ of cases. ${ }^{2,3}$

Erythema multiforme-like dermatitis is a rare skin rash that develops due to isoniazid (INH). The clinical presentation includes erythematoedematous lesions in an acral distribution with no mucosal involvement and systemic exposure to INH. Skin biopsy and patch tests are the supportive diagnostic methods. Isoniazid-associated skin rashes rarely are reported and generally are not severe enough to terminate the drug. We present a patient with psoriasis who received TB prophylaxis before antiTNF- $\alpha$ use. He presented with erythema multiforme-like dermatitis due to INH. Withdrawal of the drug and treatment of the lesions were the first steps of intolerance, followed by a patch test with the culprit drug after recovery. We discuss the diagnostic drug allergy evaluation and treatment approach.

A 37-year-old man presented with a 15-year history of severe psoriasis with frequent flares. He was treated with various topical and systemic agents including acitretin and methotrexate at 4-year intervals. Despite the addition of phototherapy, he underwent a new treatment with anti-TNF- $\alpha$, as the disease control with other treatments was insufficient. Before starting anti-TNF- $\alpha$, preventive treatment against TB with INH $(300 \mathrm{mg} / \mathrm{d})$ was indicated with $20 \mathrm{~mm}$ of purified protein derivative. On approximately the 20th day of treatment, he developed pruritic erythema with desquamation and exfoliation localized to the hands and feet (Figure 1). Isoniazid was discontinued and a topical steroid was initiated. After 3 weeks, the skin lesions were completely improved and INH was reinitiated at the same dose with antihistamine prophylaxis (oral levocetirizine $5 \mathrm{mg} / \mathrm{d}$ ). Seven days later, similar skin lesions presented that were more extensive on the arms and legs (Figure 2). Complete blood cell counts, renal and hepatic function tests, and hepatitis markers were within reference range in consultation with the allergy division.

From Kirikkale University Faculty of Medicine, Turkey. Drs. Baççıoğlu and Kalpaklıoğlu are from the Department of Pulmonary Diseases, Division of Immunology and Allergy, and Dr. Karabulut is from the Department of Dermatology.

The authors report no conflict of interest.

Correspondence: Ayşe Baççığlu, MD, Kirikkale University Faculty of Medicine, Department of Pulmonary Diseases, Division of Immunology and Allergy, Kirikkale Turkey 9071100 (aysebaccioglu@gmail.com).

doi:10.12788/cutis.0243 
To distinguish the lesions from a psoriasis attack, a punch biopsy of the eruptive dermatitis showed erythema multiforme-like dermatitis including dermal edema and perivascular lymphocytic infiltration with no relation to psoriasis but consistent with a drug eruption. Isoniazid was discontinued, and the skin lesions resolved after
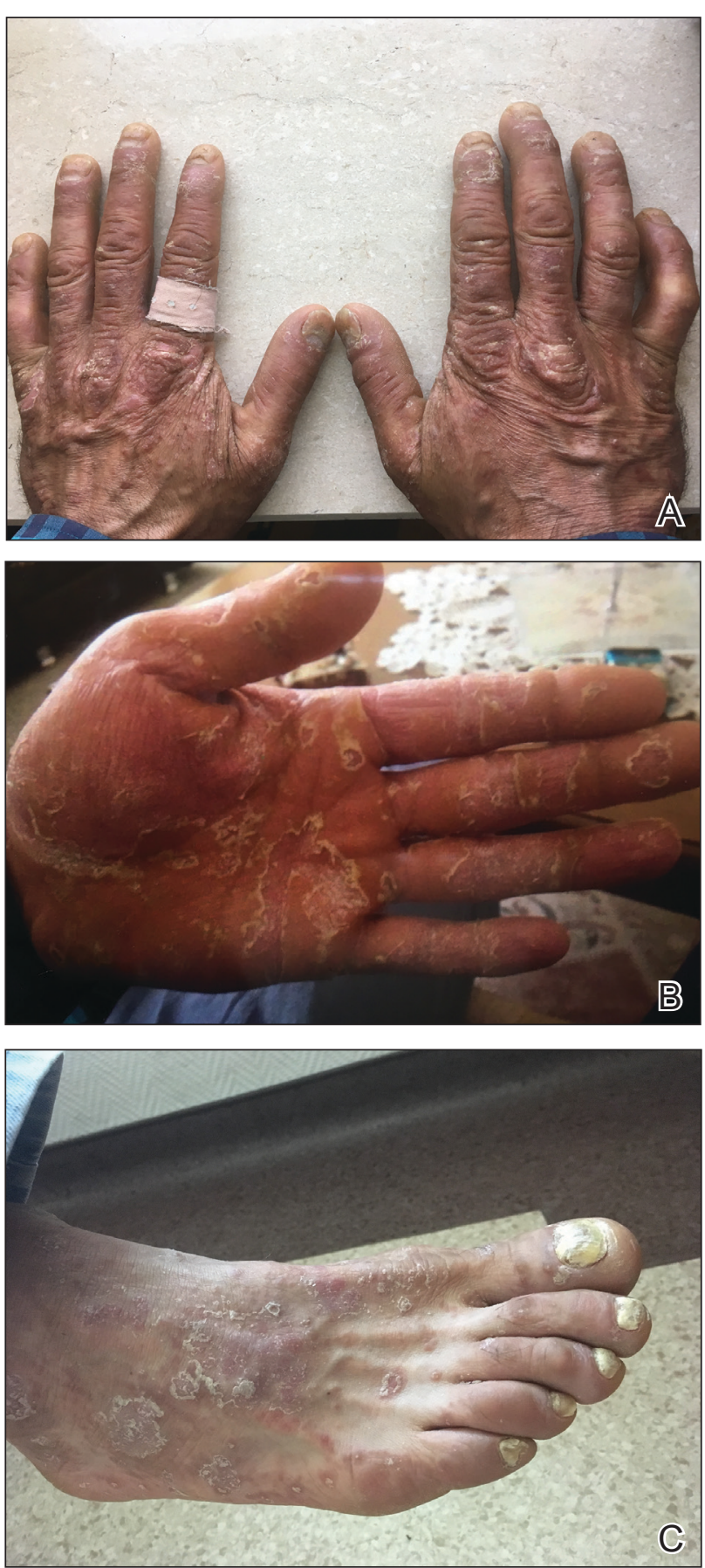

FIGURE 1. A-C, Erythema multiforme-like skin lesions developed on the hand, palm, and foot, respectively, after prophylactic isoniazid was administered prior to anti-tumor necrosis factor $\alpha$ therapy for psoriasis.
4 weeks of topical steroid and oral antihistamine use (Figure 3). There was no other drug use except INH, and a skin patch test with INH was positive at 72 hours (Figure 4). Skin tests with INH were done to 5 healthy lesions that were negative. Finally, TB prophylaxis was performed with rifampicin $(10 \mathrm{mg} / \mathrm{kg} / \mathrm{d}[600 \mathrm{mg} / \mathrm{d}])$ for 4 months with no ADRs. The patient's psoriasis lesions improved with anti-TNF- $\alpha$ that was initiated 1 month after starting TB prevention with rifampicin.

This case of erythema multiforme-like dermatitis was diagnosed with acral involvement, a positive patch test to INH, and lymphocytic inflammation in a skin biopsy. It was a drug-induced reaction, as skin lesions developed during INH intake and improved after drug withdrawal.

Isoniazid, also known as isonicotinylhydrazide, is an oral antibiotic used for the treatment of $\mathrm{TB}$ and other mycobacteria. Protective treatment against latent TB primarily is done with daily INH for 6 or 9 months; alternatively, INH may be taken weekly with rifapentine for 3 months or daily with rifampicin for 4 months. Daily rifampicin alone for 4 months also is an option. In general, these regimens have similar efficacy; however, in terms of safety, the rifampicin and rifapentine combination regimens have fewer hepatotoxicity events compared to the INH alone regimen, but there are more cutaneous and flulike reactions and gastrointestinal intolerance. ${ }^{4}$ Cutaneous ADRs to TB treatment such as mild itchiness and cutaneous eruptions usually are observed within 2 months of drug initiation. Pyrazinamide was reported as the most common drug associated with cutaneous ADRs, and INH was the rarest offending drug. ${ }^{5}$

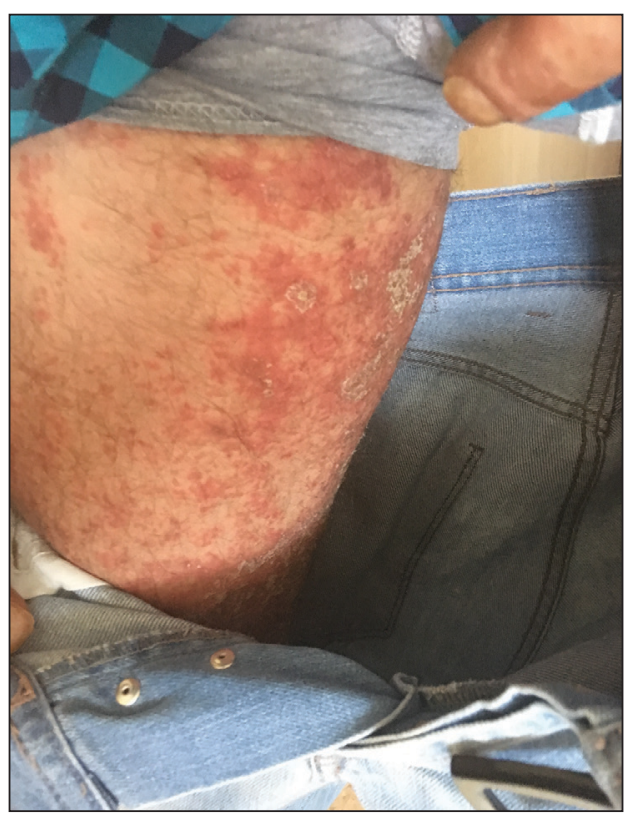

FIGURE 2. More extensive lesions developed inside the upper legs 7 days after isoniazid was readministered. 


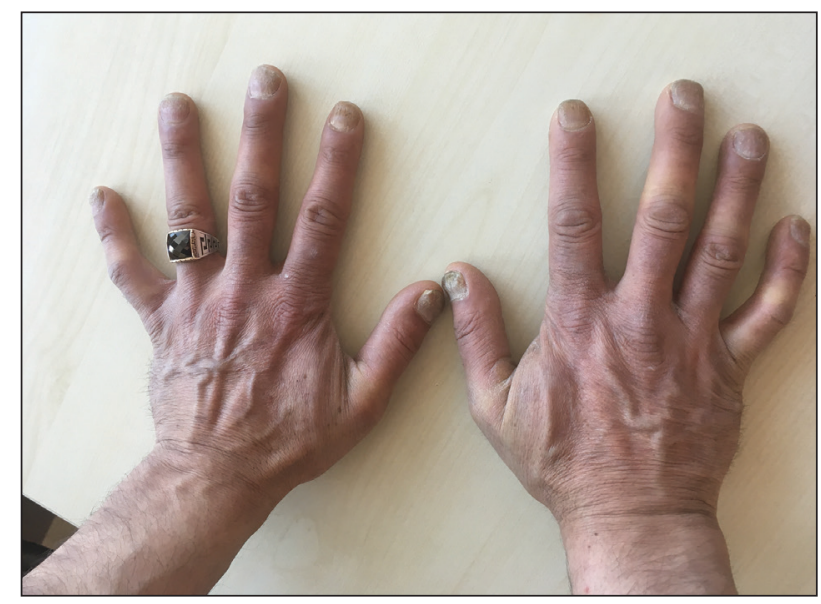

FIGURE 3. Four weeks after isoniazid was withdrawn and symptomatic treatment was initiated, improvement was seen in skin lesions on the hands.

The frequency of ADRs to INH is approximately $5.4 \%$, and the most prevalent ADRs include asymptomatic elevation of serum liver enzyme concentrations, peripheral neuropathy, and hepatotoxicity, and skin lesions are less common. ${ }^{2}$ Our patient's laboratory test results excluded vitamin B deficiency, hepatic and renal dysfunction, and neuropathy.

Previously reported skin reactions related to $\mathrm{INH}$ were late-type reactions such as maculopapular rash, dermatitis, erythema multiforme, drug reaction with eosinophilia and systemic symptoms (DRESS) syndrome, Stevens-Johnson syndrome, and toxic epidermal necrolysis. ${ }^{5,6}$ The concerning prediagnosis of psoriatic exacerbation in our patient was ruled out by the absence of typical skin lesions such as well-defined, erythematous plaques and pustules and atypical localization such as the dorsal hands and feet rather than the knees, elbows, lumbosacral region, scalp, and abdomen, which is typical of psoriasis. DRESS syndrome was unlikely with the absence of fever, lymphadenopathy, hypereosinophilia, leukocytosis, and renal and hepatic dysfunction. ${ }^{7}$ There were no widespread blisters, epidermal detachment, or mucosal involvement on the trunk or face typically associated with Stevens-Johnson syndrome and toxic epidermal necrolysis. ${ }^{7,8}$ A possible diagnosis of contact dermatitis was suspected with likely skin lesions as exfoliation and chapping, typical localization on the hands and feet, and positive patch test that supported sensitization to the drug. However, the patient's skin lesions were not eczematous (characterized by erythema, vesiculation, exudation, or bullous edema in the acute phase), and were not localized to areas of irritant exposure. ${ }^{3}$ In our patient, erythematoedematous lesions in an acral distribution with no mucosal involvement and systemic exposure to INH was compatible with erythema multiforme, whereas the absence of target appearance, positive patch test, and late appearance were incompatible with erythema multiforme. ${ }^{8}$

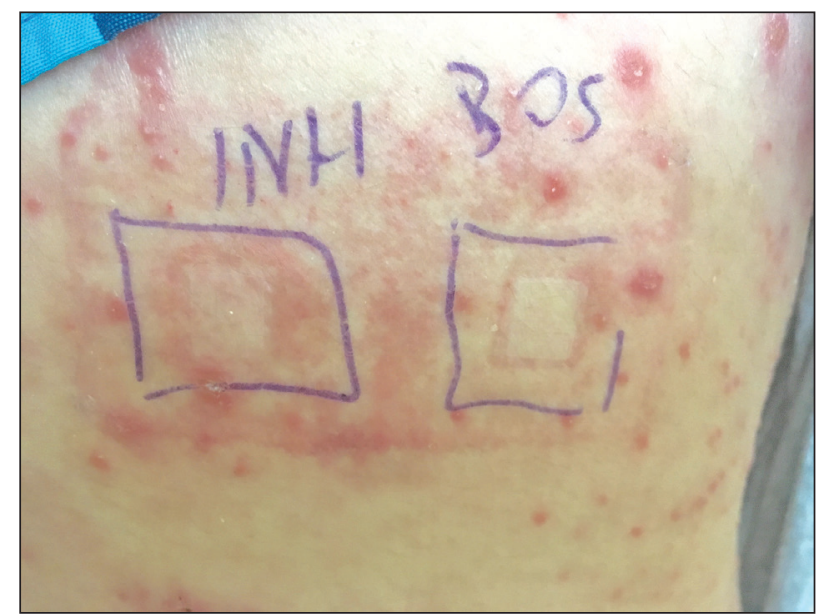

FIGURE 4. Patch test result with isoniazid (INH) and empty field (BOS) after 72 hours.

Because the clinical picture did not fit contact dermatitis or erythema multiforme, a diagnosis of erythema multiforme-like noneczematous dermatitis was suggested. Noneczematous dermatitis has subtypes that include purpuric, lichenoid, pustular, lymphomatoid, dyshidrosiform, and pigmented, as well as erythema multiforme-like contact eruptions. ${ }^{9}$ These clinical entities are not associated with contact exposure, but are related to systemic exposure, as seen in our patient. ${ }^{10}$ The patch test positivity and skin biopsy report also supported the diagnosis of erythema multiforme-like dermatitis. Erythema multiforme-like dermatitis is thought to be caused by medications or infections inducing immunocomplexes and lymphocytic infiltration in the dermis and subepidermis. Nevertheless, the prognosis was selflimiting in both. ${ }^{8}$ The clinical polymorphism caused by INH in this patient was suggested to be related with individual susceptibility, variability of contactactivating modalities, and the targeted cutaneous structures. Furthermore, among the risk factors for cutaneous ADRs-HIV, polypharmacy, older age, and preexisting renal and liver impairment-the only notable factor in this patient was psoriasis as an autoimmune disorder.

Patients with skin diseases such as psoriasis should be followed up by closer monitoring during INH use. Withdrawal of the drug and symptomatic treatment of the lesions with corticosteroid and antihistamine are the first steps of drug intolerance. After complete recovery and termination of antiallergic drugs, diagnostic tests are recommended if the drug reaction was not life-threatening. Skin prick and intradermal tests are useful in early-type drug reactions, whereas patch testing and late evaluation of an intradermal test may be helpful in the diagnosis of delayed-type reactions. The full dose of INH is avoided in an intradermal test against irritation. A patch test with INH was performed by diluting a 100-mg tablet with $1 \mathrm{~mL}$ of distilled water, and used as 1/100, 1/10, 
and 1/1 dilutions. ${ }^{8}$ Patch testing with INH also was done in 5 healthy control patients to exclude the irritation effect in this case. The rechallenge of INH was done in a controlled manner in our patient to rule out psoriasis activation since it was a localized skin reaction with no serious ADR. An oral provocation test with the culprit drug is the gold standard of drug allergy diagnosis that should be done in a tertiary hospital with an intensive care unit.

This case of erythema multiforme-like dermatitis due to INH is interesting due to systemic intake of INH, which resulted in dermatitis with localized involvement similar to erythema multiforme but with no immunologic processes or prior sensitization. With the increasing use of anti-TNF- $\alpha$ treatment, INH use will be more prevalent than in the past for the treatment of latent TB. Even though the skin-restricted ADRs of INH are rare and minor, particular attention should be paid to patients with dermatologic diseases. In our case, diagnostic drug allergy evaluation was performed to optimize the second-line treatment of $\mathrm{TB}$ infection, in addition to early withdrawal of the culprit drug.

\section{REFERENCES}

1. Vide J, Magina S. Moderate to severe psoriasis treatment challenges through the era of biological drugs. An Bras Dermatol. 2017;92:668-674.

2. Gülbay BE, Gürkan OU, Yildiz OA, et al. Side effects due to primary antituberculosis drugs during the initial phase of therapy in 1149 hospitalized patients for tuberculosis. Respir Med. 2006;100:1834-1842.

3. Holdiness MR. Contact dermatitis to antituberculosis drugs. Contact Dermatitis. 1986;15:282-288.

4. Getahun H, Matteelli A, Abubakar I, et al. Management of latent Mycobacterium tuberculosis infection: WHO guidelines for low tuberculosis burden countries. Eur Respir J. 2015;46:1563-1576.

5. Tan WC, Ong CK, Kang SC, et al. Two years review of cutaneous adverse drug reaction from first line anti-tuberculous drugs. Med J Malaysia. 2007;62:143-146.

6. Özkaya E. Eczematous-type multiple drug allergy from isoniazid and ethambutol with positive patch test results. Cutis. 2013;92:121-124.

7. Fernando SL. Drug-reaction eosinophilia and systemic symptoms and drug-induced hypersensitivity syndrome. Australas J Dermatol. 2014;55:15-23.

8. Rebollo S, Sanchez P, Vega JM, et al. Hypersensitivity syndrome from isoniazid with positive patch test. Contact Dermatitis. 2001;45:306.

9. Sokumbi O, Wetter DA. Clinical features, diagnosis, and treatment of erythema multiforme: a review for the practicing dermatologist. Int $J$ Dermatol. 2012;51:889-902.

10. Bonamonte D, Foti C, Vestita M, et al. Nummular eczema and contact allergy: a retrospective study. Dermatitis. 2012;23:153-157. 